Document downloaded from:

http://hdl.handle.net/10251/57000

This paper must be cited as:

Ricchiuti, AL.; Barrera Vilar, D.; Nonaka, K.; Sales Maicas, S. (2014). Temperature gradient sensor based on a long-fiber Bragg grating and time-frequency analysis. Optics Letters. 39(19):5729-5731. doi:10.1364/OL.39.005729.

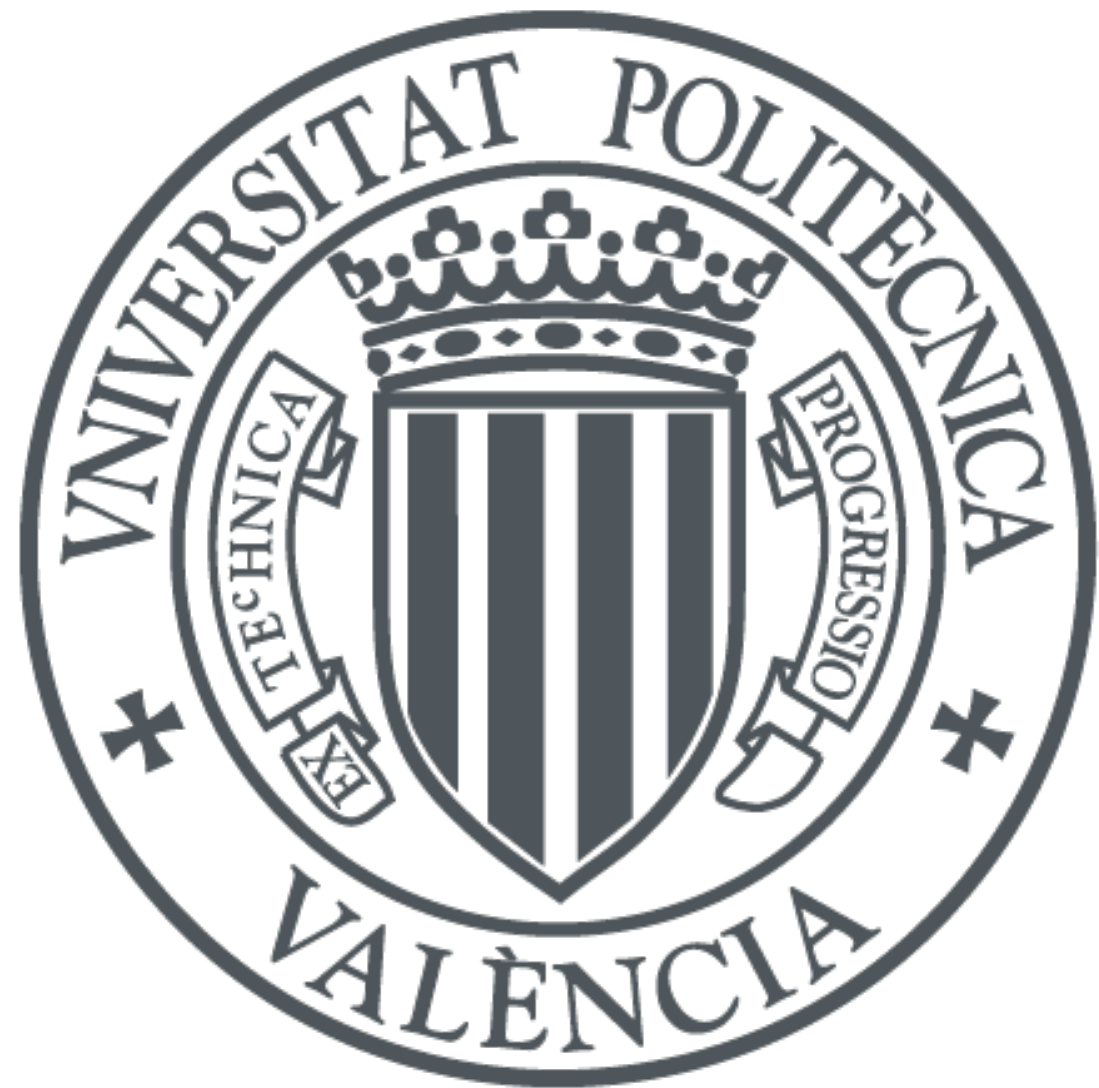

The final publication is available at

http://dx.doi.org/10.1364/OL.39.005729

Copyright Optical Society of America

Additional Information

(C) [2014 Optical Society of America.]. One print or electronic copy may be made for personal use only. Systematic reproduction and distribution, duplication of any material in this paper for a fee or for commercial purposes, or modifications of the content of this paper are prohibited. 


\title{
Temperature gradient sensor based on a long fiber Bragg grating and time-frequency analysis
}

\author{
Amelia Lavinia Ricchiuti $^{1 *}$, David Barrera ${ }^{1}$, Koji Nonaka ${ }^{2}$ and Salvador Sales ${ }^{1}$ \\ ${ }^{1}$ Optical \& Quantum Communications Group, ITEAM Research Institute, Universidad Politécnica de Valencia, \\ Camino de Vera $s / n, 46022$ Valencia, Spain \\ ${ }^{2}$ Kochi University of Technology, Tosayamada, Kami City, Kochi 782-8502, Japan \\ *Corresponding author: amricl@iteam.upv.es \\ Received July 25, 2014; revised August 27, 2014; accepted Month X, \\ XXXX; posted Month X, XXXX (Doc. ID 217827); published Month X, \\ $\mathrm{XXXX}$
}

\begin{abstract}
A photonic sensor based on a $10 \mathrm{~cm}$-long fiber Bragg grating (FBG) is presented and experimentally validated, dedicated to detect the presence and the position of a temperature gradient. The system is based on the measurement of the central frequency distribution of the grating based on time-frequency domain analysis. A short optical pulse, having duration much shorter than the transit time along the grating, is coupled into the FBG and the back-reflected pulse is scanned by means of an oscilloscope. A spatial resolution of $1 \mathrm{~mm}$, given by half the input pulse duration, is achieved. The proposed sensor is based on a simply configuration and presents a sensing range of $10 \mathrm{~cm}$, which could be further enhanced by fabricating a longer grating. () 2014 Optical Society of America

OCIS Codes: (060.3735) Fiber Bragg gratings, (060.2370) Fiber optics sensors, (280.6780) Temperature.

http://dx.doi.org/10.1364/OL.99.099999
\end{abstract}

Nowadays, fiber Bragg gratings (FBGs) have established their role as key components in all-fiber based devices due to their interesting characteristics, such as simplicity, low insertion loss, low cost, polarization independence and seamless integration in fiber optics systems. Furthermore, since FBGs are made of dielectric material, they result non-conducting, immune to electromagnetic interference (EMI), chemically inert and spark free [1]. For these reasons, FBGs have been massively implemented in different kinds of application scenarios such as sensors [2], filters [3], switches [4] and for multiplying pulse repetition rates [5], amongst others.

FBGs bring many advantages to sensing area applications over other optical fiber technologies due to their high capability for wavelength multiplexing and possibility of measuring reflected light. In this context, different methods have been proposed and demonstrated in order to interrogate the Bragg-frequency distribution along an FBG with the aim of implementing distributed temperature/strain sensors. Some of these salient techniques include optical low-coherence reflectometry (OLCR) [6], optical frequency domain reflectometry (OFDR) [7], synthesis of optical coherence function (SOCF) [8], and time-frequency analysis [9].

As far as the temperature sensing field is concerned, in previous works, high reflectivity FBGs have been used to detect hot spots [10]. Also, a temperature sensor based on a very long continuous weak FBG has been presented [11].

In this letter, a simple scheme to measure the distribution of central frequency of a $10 \mathrm{~cm}$-long FBG, based on time-frequency domain analysis is proposed and experimentally demonstrated. The measurement system is essentially based on a combination of frequency scanning of the input pulse and optical time domain reflectometry (OTDR) [12]. In fact, to perform the analysis of the Bragg-frequency distribution, a short optical pulse, having duration much shorter than the transit time along the grating, is used. The signal back-reflected from the grating is scanned by means of an oscilloscope. This way, by controlling the frequency of the interrogating pulse, the detection of a temperature gradient can be achieved, with a remarkable spatial resolution. The measurements here reported, clearly demonstrate that such a system can offer an attractive and effective solution for distributed fiber optics sensing.

The schematic diagram of the proposed FBG-based sensor is depicted in Fig. 1. A short optical pulse is launched into the long FBG through an optical circulator. The grating employed in the experiment consists in a 10 $\mathrm{cm}$-long uniform FBG with $\mathrm{a} \approx 99 \%$ reflectivity and $\mathrm{a} \approx 14$ $\mathrm{GHz}$ full-width half-maximum (FWHM). The Bragg resonance is $1553.98 \mathrm{~nm}$ at room temperature.

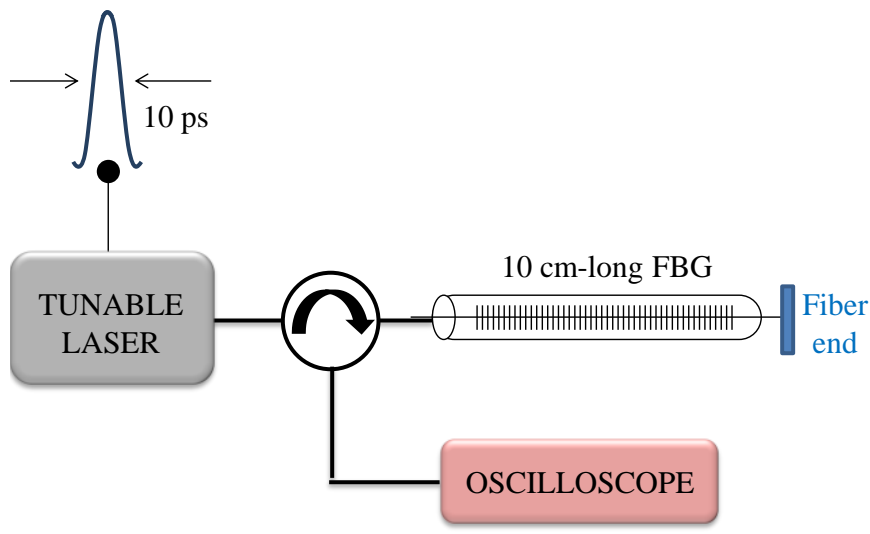

Fig. 1. Schematic diagram for interrogating the $10 \mathrm{~cm}$-long uniform FBG. 
Hence, the signal back-reflected from the grating is captured and analyzed by means of an oscilloscope. The interrogating pulse presents a duration of $10 \mathrm{ps}$ which is much shorter that the transit time along the grating. The latter can be calculated according to

$$
T_{\text {trans }}=\frac{2 n_{0} L_{F B G}}{c}
$$

being $n_{0}$ the refractive index of the fiber, $c$ the speed of light in vacuum and $L_{F B G}=10 \mathrm{~cm}$ the grating length. This way, the transit time is estimated to be $T_{\text {trans }} \approx 0.971 \mathrm{ns,}$ which is much longer than the input pulse duration.

Moreover, after the grating end a piece of single mode fiber (SMF) having length of $1 \mathrm{~cm}$ is placed. The end face of this $1 \mathrm{~cm}$-long SMF is left open in the air to provide a reflection signal that will be used as a reference point. It is worth pointing out that, even if the temperature gradient were placed at the end of the grating, the measure of its position will not be adversely affected. In fact, by using (1) the transit time in $1 \mathrm{~cm}$-long SMF is calculated to be $\approx$ 97.101 ps, resulting an order of magnitude bigger than the interrogating pulse duration.

The wavelength of the tunable laser used to perform the measurements has been previously calibrated in temperature; so as, by simply adjusting the thermistor resistance controlling the device temperature, the wavelength of the laser can be easily tuned, as depicted in Fig. 2. This way, to measure the distribution of the grating spectral properties, the central frequency of the interrogating pulse is precisely controlled by the injection current applied to the laser.

As previously mentioned, the system proposed is based on the measurement of the central frequency distribution of the FBG based on time-frequency domain analysis. When the incident pulse wavelength is far from the Bragg wavelength, at the oscilloscope only the signal related to the reflection at the end of the SMF will be retrieved, as illustrated in Fig. 3.

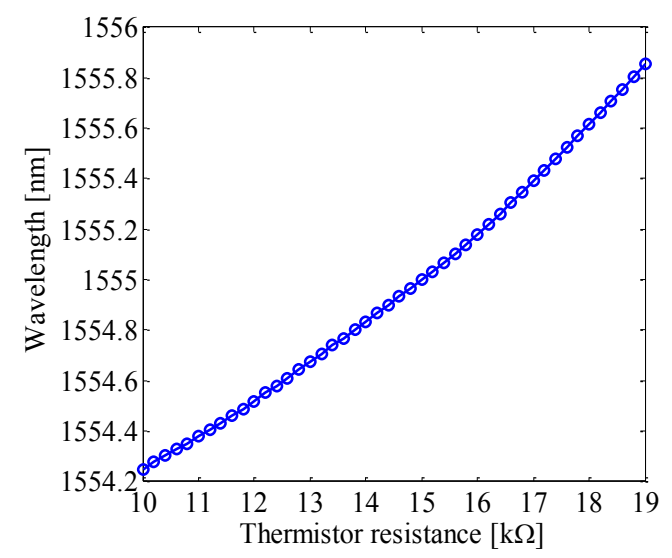

Fig. 2. Characterization of the laser wavelength tunability as function of the thermistor resistance controlling the device temperature.

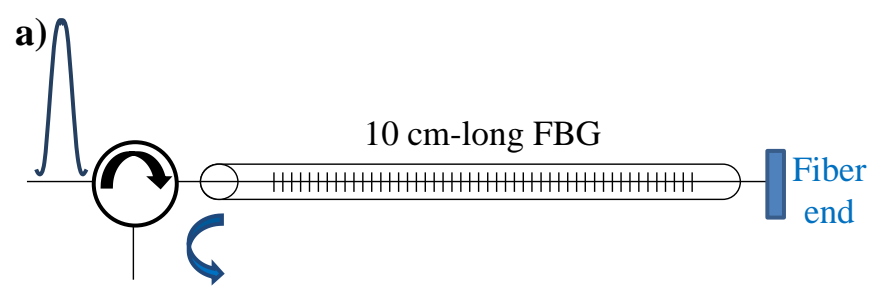

b)

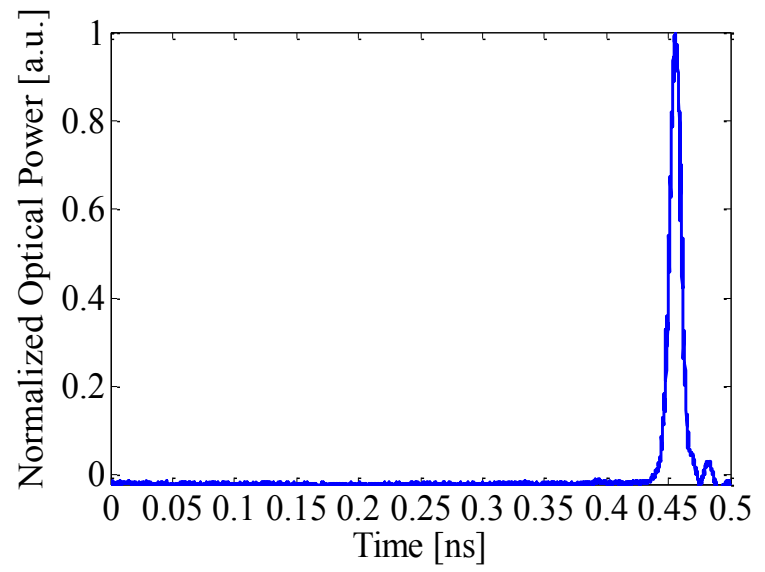

Fig. 3. a) Detail of the measurement layout showing the FBG and the reference point. b) Measurement of the reflected pulse time waveform when the interrogating pulse as a wavelength different from the Bragg wavelength. The input pulse is reflected at the end of the fiber.

Hereafter, when a hot zone is provoked along the sensing FBG, a temperature gradient is obtained. This local change of temperature will produce a local Bragg frequency shift. When this occurs, besides the signal reflected at the end of the fiber, a second reflected signal is produced (due to the refractive index changes provoked by the heating) at the point where the hot zone is located, as can be seen in the Fig. 4(a). Now, by performing a stepwise scan of the frequency of the interrogating pulse, the temperature shifts can be detected. As mentioned before, the signal reflected at the end of the grating can be used as reference point in order to obtain the exact position and distribution of the hot zone along the sensing FBG. In fact, the distance $L$ between the different temperature zones and the end of the fiber is calculated by evaluating the time separation $T$ between the reference pulse and the time waveform provoked by the hot zone according to

$$
L=\frac{T c}{2 n_{0}}
$$

This way, the temperature gradient and its distribution along the grating are calculated with a $10 \mathrm{~cm}$-long measurement range. 
a)

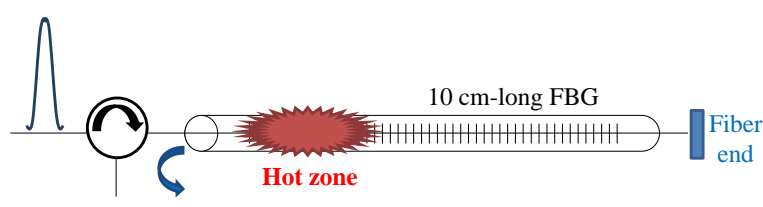

b)
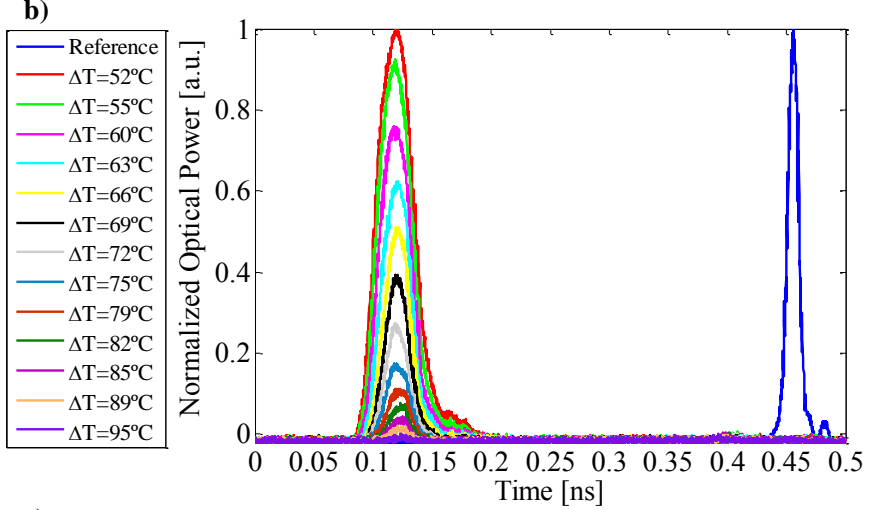

c)

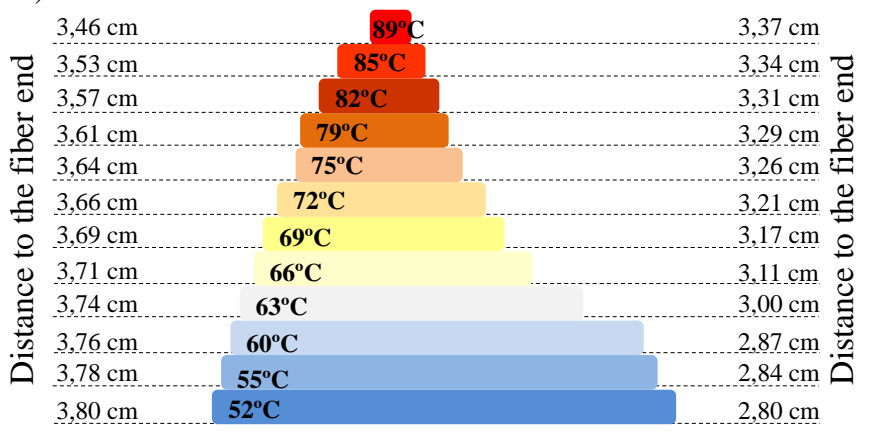

Fig. 4. a) Detail of the FBG-based temperature gradient sensor. b) Measurements of the reflected pulse time waveform as function of the laser emission wavelength. c) Characterization of the heated zone in the sensing grating. The distance of the hot zone to the end of the fiber is shown.

Figure 4(b) shows the distribution of the different temperature zones as well as their position along the grating. The hot zone has a minimum temperature of $52^{\circ} \mathrm{C}$ (see red curve in Fig. 4(a)) and a width of $1 \mathrm{~cm}$. Since the interrogating pulse wavelength is progressively increased, the width of the pulse reflected at the hot zone is gradually reduced. This fact means that the heated surface presents its highest temperature in its middle point. In fact, when the tunable laser resistance is set in order to perform an interrogating pulse wavelength of $1554.87 \mathrm{~nm}$, a temperature of $89^{\circ} \mathrm{C}$ above the room temperature can be detected. This temperature corresponds to the hottest point which is located in the midst of the hot zone (see Fig. 4(a) and 4(b)).

The spatial resolution of this technique is essentially determined by half the pulse duration, such as OTDR technique [12]. Hence, since the optical interrogating pulse has duration of $10 \mathrm{ps}$, the proposed configuration presents a $1 \mathrm{~mm}$ spatial resolution. The temperature distribution along the sensing FBG is calculated according to the fact that $10 \mathrm{pm}$ shift in Bragg wavelength correspond to $1^{\circ} \mathrm{C}$ shift in temperature. It is worth mentioning that, since the laser wavelength calibration has been performed with an accuracy of less than $5 \mathrm{pm}$, the temperature gradient measurements have been carried out with an accuracy of less than $0.5^{\circ} \mathrm{C}$.

The measurements performed simply indicate the potential of the method in term of spatial resolution and measurement range, which are certainly attractive in some research areas such as micromechanics, bio and chemical fields, among others. Nevertheless, both these values can be further enhanced by using a shorter interrogating pulse (as far as the spatial resolution is concerned), and by fabricating a longer FBG (as far as the measurement range is regarded). The initial idea beyond this experiment was to fabricate a very long FBG, but due to the limitations of our grating fabrication system, FBGs longer than $10 \mathrm{~cm}$ cannot be created today. Hence, as a proof-of-concept a $10 \mathrm{~cm}$-long FBG has been employed as a distributed temperature gradient sensor. Very long high-quality FBGs (more than $10 \mathrm{~cm}$ long) are hard to fabricate today. Nowadays, the larger size of a continuous grating that has been manufactured is over one meter. Nevertheless, no continuous FBGs having length of several tens of meters have been fabricated and demonstrated in recent works [13].

In this work, the possibility to measure the local spectral characteristics along a long uniform FBG, using optical time-frequency domain analysis has been proposed and experimentally validated. A short optical pulse, having duration much shorter than the propagation time through the grating, has been used. To perform the analysis of the Bragg-frequency distribution a technique based on a combination of frequency scanning of the input pulse and OTDR has been investigated and demonstrated. The time traces obtained by progressively incrementing the interrogating pulse wavelength as well as the reflection obtained at the end of the fiber have been used to estimate the position of the temperature gradient along the FBG.

In this way, the presence and the precise location of a local hot zone are detected with a spatial resolution of 1 $\mathrm{mm}$, dictates from half the interrogating pulse duration, which may be further improved by using shorter input pulse. The method presents a measurement range of 10 $\mathrm{cm}$, which can be enhanced by fabricating a longer sensing grating. Furthermore, as the central frequency of the signal pulse has been precisely controlled by the injection current applied to the laser, the proposed system provides stable operation and very good repeatability.

Due to the high sensitivity of the FBG spectrum to temperature and/or strain changes, the proposed measurement system has set the foundation for the development of a novel, simply and robust solution for high spatial resolution distributed fiber sensing with no need of high optical pumping and/or sophisticated controlling systems.

The authors wish to acknowledge the Infraestructura FEDER UPVOV08-3E-008, FEDER UPVOV10-3E-492, the Spanish MCINN through the project TEC2011-29120C05-05, the Valencian Government through the Ayuda Complementaria ACOMP/2013/146 and the financial support given by the Research Excellency Award Program GVA PROMETEO 2013/012. 


\section{References}

1. B. Culshaw, J. Lightwave Technol. 22, 39 (2004).

2. A. D. Kersey, M. A. Davis, H. J. Patrick, M. LeBlanc, K. P. Koo, C. G. Askins, M. A. Putnam, and E. J. Friebele, J. Lightwave Technol. 15, 1442 (1997).

3. S. Y. Li, N. Q. Ngo, S. C. Tjin, P. Shum, and J. Zhang, Opt. Lett. 29, 29 (2004).

4. H. Uno, A. Kojima, A. Shibano, and O. Mikami, "Optical wavelength switch using strain-controlled fiber Bragg gratings," Proc. SPIE 3740 (1999).

5. J. Azaña and M. A. Muriel, IEEE J. Sel. Top. Quantum Electron. 7, 728 (2001).

6. M. Volanthen, H. Geiger, and J. P. Dakin, J. Lightwave Technol. 15, 2076 (1997).

7. H. Murayama, H. Igawa, K. Kageyama, K. Ohta, I. Ohsawa, K. Uzawa, M. Kanai, T. Kasai, and I. Yamaguchi, in Optical Fiber Sensors, OSA Technical Digest (CD) (Optical Society of America, 2006), paper ThE40.

8. K. Hotate and K. Kajiwara, Opt. Express 16, 7881 (2008).

9. J. Sancho, S. Chin, D. Barrera, S. Sales, and L. Thévenaz, Opt. Express 21, 7171 (2013).

10. A. L. Ricchiuti, D. Barrera, S. Sales, L. Thévenaz, and J. Capmany, Opt. Express 21, 28175 (2013).

11. L. Thévenaz, S. Chin, J. Sancho, and Salvador Sales, Proc. SPIE 9157 (2014).

12. M. K. Barnoski, M. D. Rourke, S. M. Jensen and R.T. Melville, Appl. Opt. 16, 2375 (1977).

13. "FBGS - Draw Tower Gratings", http://www.fbgs.com/. 


\section{Full references}

1. B. Culshaw, "Optical fiber sensor technologies: opportunities and perhaps pitfalls," J. Lightwave Technol. 22, 39-50 (2004).

2. A. D. Kersey, M. A. Davis, H. J. Patrick, M. LeBlanc, K. P. Koo, C. G. Askins, M. A. Putnam, and E. J. Friebele, "Fiber grating sensors," J. Lightwave Technol. 15(8), 1442-1463 (1997).

3. S. Y. Li, N. Q. Ngo, S. C. Tjin, P. Shum, and J. Zhang, "Thermally tunable narrow-bandpass filter based on linearly chirped fiber Bragg grating," Opt. Lett. 29(1), 2931 (2004).

4. H. Uno, A. Kojima, A. Shibano, and O. Mikami, "Optical wavelength switch using strain-controlled fiber Bragg gratings," Proc. SPIE 3740 (1999).

5. J. Azaña and M. A. Muriel, "Temporal self-imaging effects: theory and application for multiplying pulse repetition rates," IEEE J. Sel. Top. Quantum Electron. 7, 728 - 744 (2001).

6. M. Volanthen, H. Geiger, and J. P. Dakin, "Distributed grating sensors using low-coherence reflectometry," J. Lightwave Technol. 15(11), 2076-2082 (1997).

7. H. Murayama, H. Igawa, K. Kageyama, K. Ohta, I. Ohsawa, K. Uzawa, M. Kanai, T. Kasai, and I. Yamaguchi, "Distributed strain measurement with high spatial resolution using fiber Bragg gratings and optical frequency domain reflectometry," in Optical Fiber Sensors, OSA Technical Digest (CD) (Optical Society of America, 2006), paper ThE40.

8. K. Hotate and K. Kajiwara, "Proposal and experimental verification of Bragg wavelength distribution measurement within a long-length FBG by synthesis of optical coherence function,” Opt. Express 16(11), 78817887 (2008).

9. J. Sancho, S. Chin, D. Barrera, S. Sales, and L. Thévenaz, "Time-frequency analysis of long fiber Bragg gratings with low reflectivity,” Opt. Express 21(6), 7171-7179 (2013).

10. A. L. Ricchiuti, D. Barrera, S. Sales, L. Thévenaz, and J. Capmany, "Long fiber Bragg grating sensor interrogation using discrete-time microwave photonic filtering techniques," Opt. Express 21(23), 28175-28181 (2013).

11. L. Thévenaz, S. Chin, J. Sancho, and Salvador Sales, "Novel technique for distributed fibre sensing based on faint long gratings (FLOGs)", Proc. SPIE 9157 (2014).

12. M. K. Barnoski, M. D. Rourke, S. M. Jensen and R.T. Melville, "Optical time domain reflectometer", Appl. Opt. 16(9), 2375-2379 (1977).

13. "FBGS - Draw Tower Gratings", http://www.fbgs.com/. 